\title{
LA EPISTEMOLOGÍA DE LAS CIENCIAS SOCIALES EN LA FORMACIÓN POR COMPETENCIAS DEL PREGRADO
}

\section{SOCIAL SCIENCE EPISTEMOLOGY IN GRADUATE COMPETENCY-BASED TRAINING}

Mg. Fernando Farías (ffarias@ubiobio.cl) Escuela Trabajo Social, Universidad del Bío-Bío (Concepción, Chile)

\begin{abstract}
The article discusses the role of epistemology in the training and professional skills of social scientists in the new context of higher education, which appears to force the idea of a competency-based training, through which its required an articulation of the cognitive, procedural and attitudinal elements. The need for epistemological training is to be understood as a process beyond itself, as a unit of learning that generates ways of thinking, such as analytical thinking, synthetical, critical, among others, as well as procedures used for the practice, and finally, in the area of attitudinal competencies, it is possible to recognize an epistemology of pluralism and tolerance characteristic of contemporary societies.
\end{abstract}

Keywords: professional skills, higher education, competency-based training, pluralism.

\section{Resumen}

El artículo analiza, el papel que juega la epistemología en la formación profesional y disciplinaria de las ciencias sociales en el nuevo contexto de educación superior, en donde, aparece con fuerza la idea de una formación basada en competencias, a través de la cual se exige una articulación de los elementos cognitivos, procedimentales y actitudinales en la formación de las nuevas generaciones de profesionales de éstos campos disciplinarios. De este modo la necesidad de una formación epistemológica se ha de entender como un proceso que más allá de los conocimientos propios y necesarios de éste campo disciplinario, se la debe pensar como aquella unidad de aprendizaje que tributa a generar formas de pensar, tales como un pensar analítico, sintético, crítico, entre otros; así también como procedimientos que sirvan para el ejercicio profesional, lo cual se evidencia por medio de la vigilancia epistemológica; finalmente, en el ámbito de las competencias actitudinales, es posible reconocer en la epistemología una contribución al pluralismo y la tolerancia propio de las sociedades contemporáneas.

Palabras clave: competencias profesionales, educación superior, competencias, pluralismo.

\section{Nota}

Proyecto de Investigación financiado por la Dirección de Investigación de la Universidad del Bío-Bío (Proyecto DIUBB № 080524 1/I) 
Todas las teorías son legítimas y ninguna tiene importancia. Lo que importa es lo que se hace con ellas.

J.L. Borges

\section{Introducción}

El conjunto de profesiones y disciplinas que hoy denominamos de las ciencias sociales tienen su origen histórico en los albores del siglo XIX como consecuencia de los procesos que experimentaba la sociedad europea de consolidación de los Estados modernos, el capitalismo y la industrialización. En este contexto las nacientes disciplinas se intentaban consolidar a través de su legitimidad por medio de su vocación a contribuir a mermar los problemas que presentaban sus sociedades, producto del contexto de cambios y transformación. Para la consecución de tal propósito se hizo necesario organizar y racionalizar los cambios sociales a partir de las concepciones de ciencia en ese entonces imperantes, a saber las ciencias físiconaturales. Como resultado se tuvieron un conjunto de conocimientos "objetivos" a partir de la información empírica (Wallerstein 1997). Empero, las concepciones epistemológicas adoptadas por estas nuevas disciplinas intentaron emular a las ciencias experimentales, generando en un primer momento de su desarrollo una visión restringida de su quehacer y su cientificidad.

Tal concepción de ciencia, a la cual estos nuevos campos disciplinarios orientaron su quehacer, no estuvo exenta de polémicas desde sus inicios. Basta señalar las reyertas intelectuales que se generó entre la concepción de ciencia social francesa y alemana, representada en los conceptos ya clásicos de naturwissenschaften versus gesitesswissenchaften, en donde el objeto de estudio de estas nuevas disciplinas no es externo al hombre, sino el medio en el que el hombre está inserto, lo cual provoca que el investigador social pueda captar el mundo que investiga desde dentro, pues el investigador es parte del mismo mundo que investiga (Dilthey 1986). Lo anterior exige la necesidad que estas nuevas disciplinas se emancipen de una epistemología y metodología de las ciencias físico-naturales. Como otro elemento significativo de tal polémica se encuentra la distinción entre una ciencia nomotética e ideográfica (Rickert), y la distinción entre una ciencia explicativa versus comprensiva (Weber). Tal polémica se encuentra bien documentada en la tesis propuesta por G. H. von Wright, entre las concepciones de ciencia Aristotélica y Galileana. En síntesis, tal polémica se trata que los procedimientos científicos de las ciencias naturales (métodos, técnicas o instrumentos de medición) no fueran pertinentes para el estudio de las realidades histórico-sociales y culturales.

En la actualidad tal polémica se encuentra representada sintéticamente entre los conceptos de Metodologías Cuantitativas versus Cualitativas, y a la reducción casi absurda entre cantidad y cualidad. Sin embargo, desde hace ya unos años se he intentado superar tal contradicción, postulando a posiciones pseudoconciliatorias entre las metodologías cuantitativas y cualitativas, en donde se plantea una integración a partir de sus propias opciones epistemológicas. Ejemplos de tal "integración" se observa en Nagel (1981) en donde propone la necesidad de una ciencia unificada, a partir de la concepción cuantitativa de la ciencia. De igual modo, pero desde la concepción cualitativa se aprecia la propuesta de “integración” de Znaniecki, el cual insiste en la distinción del objeto de estudio entre las ciencias sociales y las físico-naturales. Sin embargo esta antinomia desaparecería una vez que la concepción cuantitativa acceda a reconocer que los datos sociales o culturales proceden de alguien y nunca de nadie. Otra de las propuestas conciliatorias entre métodos cuantitativos y cualitativos fue lo planteado por la Escuela de Frankfurt, aunque desde una perspectiva crítico-dialéctica, que apela a la posibilidad de integración entre la explicación y comprensión. Es 
así que Adorno (1973) sostiene la idea que la totalidad conceptual está compuesta por lo idéntico y diferente, y esta se encuentra unida por la unión y contradicción de la dialéctica.

En la actualidad existen tímidos intentos por correlacionar ambos procedimientos, al menos en el plano teórico-epistemológico, debido al profundo arraigo de los paradigmas en la comunidad de investigadores sociales. Sin embargo, desde el plano de la investigación empírica, es posible entrever de modo más claro la unidad de dichos procedimientos o complementación de los paradigmas cuantitativos-cualitativos. La integración metodológica se aprecia con mayor claridad a través de la compatibilidad de métodos y/o técnicas en el proceso de investigación empírica (Briones 1994), antes que en una integración de paradigmas. Sin embargo, al intentar buscar una justificación epistemológica a tal propuesta, es posible encontrarla en lo que se denomina el pluralismo epistemológico de Feyerabend.

Para Feyerabend (1991), la práctica científica es un proceso social en donde los investigadores intentan resolver problemas, y para ello, éstos, han de buscar diferentes estrategias conformes y coherentes a la naturaleza de la realidad que investigan o intentan resolver. Es por ello que se vuelve irrelevante mostrar la superioridad de un paradigma sobre el otro, dado que al ser estilos cognitivos diferentes (Toledo 1998) la superioridad de uno sobre otro ya está determinada por el punto de vista que se tiene para observar la realidad; en tanto, el estilo cognitivo cuantitativo o cualitativo, posee sus criterios de inclusión/exclusión de información, definición de realidad, procedimientos o estrategias de acceso y análisis de la información que define como relevante. Por lo señalado anteriormente, la cuestión está en la búsqueda permanente de procedimientos que resuelvan los problemas, al decir del mismo Feyerabend (1978:5): “No hay ningún único procedimiento o conjunto de reglas que sea fundamental en toda investigación y garantice que es científica $y$, por consiguiente, digna de crédito. Todo proyecto, toda teoría o procedimiento ha de ser juzgado por sus propios méritos y de acuerdo con criterios que se adecuen al proceso en cuestión".

De lo anterior se desprende que la investigación no se encuentra limitada a estándares generales (metodologías cualitativas o cuantitativas y/o paradigmas), sino más bien ha de responder con pertinencia de sus procedimientos a la situación, a fin de resolver con éxito el fenómeno u hecho que investigue. Para ello se utilizan todas las estrategias técnicas y metodológicas disponibles en la historia de las ciencias sociales, para alcanzar los objetivos trazados con los más óptimos niveles de evidencia y certeza que se puedan alcanzar. Bajo este ideario, basta recordar las palabras de Cook y Reinchardt (1986:23) cuando señalan: "tratar como incompatibles a los tipos de métodos estimula obviamente a los investigadores a emplear un solo uno u otro cuando la combinación de los dos sería más adecuada para las necesidades de la investigación. Paraliza así mismo cualquier tentativa de superar las diferencias entre las partes enfrentadas en el debate acerca de los mismos métodos".

En síntesis, cuando la discusión parte desde la práctica de la investigación, la preeminencia de uno u otro paradigma se vuelve inocua, pues la pertinencia de las estrategias metodológicas se resuelven en función de la pregunta: ¿cómo se resuelve de mejor manera el problema de estudio que se esté abordando? En consecuencia, el problemas de los "dogmatismos" epistemológicos y metodológicos se desvanece y la epistemología recobra su función en la investigación social, que a nuestro modo de ver se encuentra en la vigilancia de la coherencia, lógica, validez y confiabilidad de los resultados obtenidos tras el proceso de investigación. De lo anteriormente señalado, es que las ciencias sociales son llamadas disciplinas multiparadigmáticas, en el sentido que los problemas que aborda no pueden ser limitados a un lenguaje único con su consecuente modo de aprehensión de dicha realidad. 
En consecuencia, cuando se sostiene que la epistemología está al servicio de la investigación, significa no anteponer los dogmatismos, los estilos cognitivos o preferencias metodológicas en la práctica de la investigación empírica, sino, realizar una vigilancia epistémica, en el sentido de un examen exhaustivo y permanente a las decisiones tomadas en el proceso de la investigación o de resolución de problema para el cual fue realizado el estudio. En otros términos, se trata de llevar a cabo una investigación epistemológicamente fundada, de tal modo que libere a los investigadores de los reduccionismos metodológicos y reconocer la multidimensionalidad de la realidad social. Es ahí donde la epistemología desempeña su papel procedimental, y no en la mera justificación de las decisiones previamente adoptadas.

Bajo el concepto de vigilancia epistemológica acuñada por Bachelard (1991), así como por Canguilhem y Koyré en el contexto del espíritu científico de una época, se entiende por tal la necesidad de corrección del conocimiento y ampliación de los esquemas del saber, dado que todo conocimiento posee su historia y esquemas variables en su proceso de construcción. Es por ello que tal idea no se reduce a una vigilancia intelectual o de sus métodos; sino más bien, es una forma de reflexión acerca de los modos de producción de conocimientos, en donde se pone a prueba las certidumbres racionales, teóricas, metodológicas, así como las mismas interpretaciones socialmente aceptadas. Bajo esta misma idea serán Bourdie y Wacquant (1995:33) quienes especifican la función de la vigilancia epistemológica cuando señalan: "la exploración sistemática de las categorías de pensamientos no pensados -en tanto objetivados e inscritos como esquemas de percepción y apreciación- que delimitan lo pensable y predeterminan el pensamiento y que guían la realización de la práctica del trabajo de investigación".

En síntesis, la vigilancia epistemológica apunta a develar el por qué y el cómo se piensa como se piensa. En estos mismos términos, pero bajo otra concepción, se encuentra el concepto de observación de segundo orden aportado de la teoría de sistemas cerrados de Luhmann.

De igual modo, la vigilancia epistemológica contribuye a derrumbar la antinomia entre paradigmas cualitativo (subjetivismo) y cuantitativo (objetivismo), en tanto, al centrar su examen en la construcción del conocimiento, revela las posiciones teóricas y epistemológicas subyacentes que condicionan los conceptos fundamentales y el objeto mismo del conocimiento, así como sus métodos de investigación y sus efectos o resultados.

\section{Formación por Competencias y Epistemología}

La formación universitaria ha de desarrollar una enseñanza en los ámbitos de lo Profesional y Disciplinario, el primero de ello directamente relacionado con el saber-hacer orientado al bien común propio a las profesiones, y/o con la demanda del mundo del trabajo, por lo requerido tanto por empleadores o usuario de los servicios profesionales; en cambio, la dimensión disciplinaria, se asocia al campo de la producción de conocimientos (el saber), de este modo Morin (1999) nos señala que la disciplina corresponde a una categoría organizadora dentro del conocimiento científico, inserto dentro de un conjunto científico más amplio, con su autonomía, por medio de la cual establece su frontera disciplinaria, con su lenguaje propio, sus métodos y técnicas, y sus teorías.

Tal distinción se encuentran en una constante interacción, pues, el saber trasforma el hacer y el hacer al saber, y parafraseando a Lewin, no hay nada más práctico que una buena teoría. Empero, la formación profesional y disciplinaria no se limita exclusivamente a una exclusiva instrucción tecnocrática, sino también 
se encuentra transversalmente orientada con actitudes o valores necesarios para el ejercicio de la profesión y desarrollo de la disciplina.

En las últimas décadas se ha introducido con fuerza el enfoque por competencias en los sistemas de educación superior, tanto a nivel nacional como internacionalmente, esto con la finalidad de desarrollar una serie de destrezas y habilidades específicas en las nuevas generaciones de profesionales, para un mejor desempeño en los nuevos contextos laborales. De este modo, la formación por competencias es el proceso de enseñanza-aprendizaje que transfiere conocimientos, habilidades y actitudes, capacitando a los estudiantes para movilizarlos en diferentes contextos laborales.

La actual perspectiva en la educación superior exige a las nuevas generaciones de profesionales conocimientos acabados, no solo en el ámbito específico de su profesión-disciplina, sino de cuestionamientos fundamentales y críticos para el desarrollo de los ejercicios profesionales.

En la comunidad académica existe un amplio acuerdo que las competencias profesionales involucra tres dimensiones, o aspectos de un mismo fenómeno, a saber: (1) Competencias Cognitivas, las cuales son fundamentalmente los conocimientos disciplinarios, cuyo centro está en el saber comprender, analizar, relacionar y sintetizar ciertos conocimientos, fenómenos o sistemas. (2) Competencias Procedimentales, son aquellas que permiten saber qué hacer en determinadas situaciones profesionales; es decir, orientados a generar ciertos productos. (3) Competencias Actitudinales, aquellas referidas a aspectos volitivos o éticos que orientan y otorgan sentido al saber y al hacer.

En consecuencia, de lo anteriormente señalado se infiere que la formación por competencias no se limita a una formación orientada exclusivamente hacia el hacer, sino del saber-hacer, en consecuencia, lo teórico, lo procedimental y actitudinal deben estar armónicamente considerados en los procesos de enseñanzaaprendizaje. Frente a lo anterior, cobra relevancia la pregunta ¿cuál es papel que cumple en la formación profesional por competencias la epistemología de las ciencias sociales?, o bien, ¿para qué sirve la epistemología en la formación profesional de las ciencias sociales en el contexto de una formación por competencias?, ¿qué competencias desarrolla la epistemología?

De acuerdo a la clasificación de competencias adoptadas, la epistemología contribuye al desarrollo de competencias instrumentales cognitivas y metodológicas, durante el proceso de formación profesional y disciplinaria, habilitando a las nuevas generaciones de profesionales de las ciencias sociales para un ejercicio profesional y disciplinario competente en diferentes contextos.

La reflexión epistemológica cobra importancia en el plano disciplinario de las ciencias sociales, como se ha señalado en el primer acápite de este artículo, en tanto es una reflexión sobre la ciencia que contribuye a vigilar los nuevos conocimientos, sus fundamentos, su justificación, su posibilidad, sus procesos, sus resultados, su papel en la sociedad y la cultura (Toledo 2004). Estos aprendizajes se vuelven necesarios debido que el pensamiento científico no se reduce a un "recetario", llamado método científico, olvidando los aspectos socioculturales y cognitivos que éste desarrolla. Según Lakatos (1981:338), "la formación científica -atomizada de acuerdo con técnicas distintas y separadas- ha degenerado en entrenamiento científico. No hay que sorprenderse de que ello desanime a las mentes críticas". Además de los conocimientos y actitudes que la epistemología proporciona para el desarrollo disciplinario, se hace necesario en el actual contexto de la educación superior examinar las competencias que genera la epistemología tanto para el ejercicio profesional como disciplinario. Lo anterior, producto que los 
aprendizajes de la epistemología se orientan más allá que un recetario de métodos y lógicas o, a la simplemente repetición de autores con sus respectivas teorías, sino más bien, es indispensable puesto que enseñan a pensar.

De acuerdo a lo señalado anteriormente, la epistemología como unidad de aprendizaje inserta en el proceso de formación de los profesionales de las ciencias sociales, tributa al desarrollo de una serie de competencias que van más allá de los exclusivos conocimientos disciplinarios (aunque siguen siendo fundamentales), como son los diferentes tipos de pensamientos que contribuyen a una vigilancia epistémica: Análisis, Síntesis, Crítica, Creatividad, Reflexividad, Lógica, Analógico, Práctico, Deliberativo, Resolución de Problemas.

A continuación se presentan los diferentes estilos de pensamiento que tributa la epistemología como unidad de aprendizaje en la formación profesional y disciplinaria de las ciencias sociales:

Pensamiento Analítico, entendido como la capacidad del pensar que permite establecer diferencias y/o separar las partes de un todo hasta llegar a conocer sus principios o elementos que lo constituyen, a fin de establecer las relaciones entre los elementos internos a un problema.

Pensamiento Sintético, por la cual se entiende la capacidad que permite organizar e integrar los componentes previamente conocidos a través del análisis, para luego interrelacionarlo para formar un todo. En consecuencia, es aquel tipo de pensar que nos permite comprender y afrontar la realidad mediante patrones globales. Una vez que se conocen los diversos elementos que componen a la ciencia -lo analíticose está en condiciones de integrar y elevar el nivel de comprensión.

Pensamiento Crítico, entendido como la capacidad del pensar que cuestiona las cosas y se interesa por los fundamentos en los que se asientan las cosas, acciones, juicios, tanto propios como ajenos. Es aquel tipo de pensar que duda de las certezas, de lo único y lo absoluto, desafiando el consenso para alcanzar respuestas diferentes a las que se imponen en un determinado consenso.

Pensamiento Creativo, entendido como aquellos procesos del comportamiento mental que genera procesos de búsqueda y descubrimiento de soluciones nuevas y novedosas, con sentido y apropiadas al mismo, en los diferentes ámbitos de la vida.

Pensamiento Reflexivo, capacidad del pensamiento que facilita el reconocimiento y el desarrollo de los modos de pensar que utilizamos en la resolución de algún problema o en la realización de alguna tarea.

Pensamiento Lógico, comportamiento mental que desarrolla las formas de pensar propias del conocimiento en general y del conocimiento científico en particular, dedicando su atención a la estructura del mismo. La importancia del desarrollo de este pensamiento en la estructura mental de los profesionales es que permite comprender tanto la importancia y estructura tanto de la lógica inductiva como deductiva en el proceso de investigación.

Pensamiento Analógico, capacidad del pensamiento para realizar comparaciones entre fenómenos que mantienen una cierta semejanza a nivel funcional o estructural, a fin de lograr establecer relaciones de semejanza o similitud entre cosas diferentes. 
Pensamiento Práctico, a pesar que se piensa que la epistemología es una disciplina eminentemente teórica, la epistemología estimula la competencia cognoscitiva de reflexionar antes de seleccionar el curso de acción de mayor pertinencia, atendiendo a la información disponible y a establecer el proceso a seguir para alcanzar los objetivos con eficacia y eficiencia. De este modo, dicho pensar se encuentra dirigido a la acción, en el sentido de prácticas reflexivas. De ahí la importancia de la epistemología en tanto tributa a la motivación en las nuevas generaciones de profesionales a reflexionar sobre las acciones que deberán realizar en su actuar laboral.

Pensamiento Deliberativo, manera de pensar que reflexiona en relación a los pros y contras de nuestras decisiones antes de adoptarlas y examina la razón o sinrazón de los puntos de vistas antes de emitir un juicio, o tomar una acción. El desarrollo de tal forma de pensar cobra vital importancia, pues, la acción misma de la investigación corresponde a una permanente toma de decisiones, respecto a la pertinencia de determinadas metodologías o diseños, de acuerdo al fenómeno que se encuentre investigando; así como, al dar las razones, no solo pragmáticas sino también teóricas de tales opciones.

Pensamiento Resolución de Problemas, esta competencia se realiza aplicando los pensamientos antes descritos; pero centrándose en la capacidad de llegar a una solución fundamentada y efectiva, es decir, teniendo claridad en el modo de llegar a la meta, que en nuestro caso es la resolución de problemas de investigaciones sociales. Para la activación de ésta competencia es necesaria la identificación de la situación, el análisis y síntesis en tanto definición de elementos significativos que componen el problema, así como su comprensión (cuál es el ideal de solución); su lógica, en tanto identificar interrelaciones y procedimientos metódicos que permitan acercarse a su solución y ejecutar y validar los resultados obtenidos.

Con relación a las competencias actitudinales -saber estar en el mundo- que desarrolla la epistemología de las ciencias sociales, y bajo la apertura que nos entrega la propuesta de Feyerabend, que no se restringe exclusivamente a la dimensión epistemológica-metodológica, sino que también nos presenta una dimensión ético-política como lo demuestra Facuse (2003); es posible apreciar que las competencias actitudinales a las cuales tributa la epistemología se encuentra el pluralismo ético-político, el cual niega la idea de verdades universales, en tanto, el mismo concepto de verdad es una noción relativa, el cual dependerá siempre de la ontología de la cual proceda, es por ello que además de los saberes propios que otorga estas disciplinas, contribuye a la formación de ciudadanos/as que estimulan la democracia con tolerancia a otras visiones, ideologías y perspectivas.

\section{Conclusiones}

El tránsito que han experimentado las profesiones y disciplinas de las ciencias sociales, a partir del siglo XIX, hasta su actual estado, nos permite visualizar un recorrido que parte de la emulación de procedimientos metodológicos exógenos a la naturaleza de sus fenómenos u objetos de investigación, pasando posteriormente por estrategias de validación de sus criterios de cientificidad a través de la negación de todo lo análogo a las ciencias físico-naturales, generando de este modo posiciones irreconciliables al interior de la misma comunidad de científicos sociales entre paradigmas o metodologías, como ha sido, la distinción entre lo cualitativo versus lo cuantitativo. 
Será a partir de los aportes de epistemólogos como Kuhn y Feyerabend, en donde la investigación social encontrará fundamentos para una visión pluralista y menos dogmática en sus procedimientos metodológicos; en donde prima la resolución de problemas teóricos por sobre las anquilosadas estructuras mentales de antagonismo entre las metodologías cualitativas como cuantitativas. Tras estos mismos aportes, es que cobra relevancia la vigilancia epistemológica que debe acompañar la investigación empírica, puesto que en la realidad social, al ser una realidad pluridimensional, no existen procedimientos estables y útiles frente a toda situación, sino, más bien que frente a cada problema de estudio, los procedimientos deben ser adecuados a sus respectivos contextos.

La formación de nuevos profesionales de las ciencias sociales en el contexto de una educación por competencias, exige a la epistemología tributar más allá de los conocimientos propios de su campo disciplinario, sino, más bien, exige contribuir a desarrollar una forma de pensamiento que sea capaz de movilizar en diferentes contextos profesional-disciplinario, de manera armónica y coherente a las tres dimensiones de las competencias. En este sentido, la epistemología al interior de un plan curricular de pregrado tributa a una serie de competencias cognitivas, procedimentales y actitudinales, que van más allá de los conocimientos propios del campo disciplinario de la filosofía de las ciencias sociales; dado que a través de sus unidades de aprendizaje (contenidos) de los programas de epistemología, contribuye al desarrollo de competencias genéricas cognitivas antes descritas, y juntos a estas, el desarrollo de una actitud crítica, de búsqueda y apertura de nuevos conocimientos con su respectiva rigurosidad y compromiso con el saber epistémicamente fundado. En el ámbito de los procedimientos se observa que a través de los programas de epistemología, se estimula el desarrollo de las capacidades conocidas como vigilancia epistemológica, en tanto, contribuye a formar las habilidades de formular preguntas al proceso mismo del conocimiento, a su lógica, a la naturaleza del objeto que investiga, a los procedimientos metodológicos y a la validez misma del conocimiento producido. Finalmente, en el ámbito de las competencias actitudinales, la importancia de la epistemología es que contribuye a la construcción de ciudadanos pluralistas, tolerantes, críticos y abiertos a las otras formas de saber así como a la aceptación de la diversidad social y cultural de las sociedades contemporáneas.

\section{Bibliografía}

Adorno, T. 1973. La disputa en el positivismo en la sociología alemana. Barcelona: Grijalbo.

Bachelard, G. 1991. La formación del espíritu científico: contribución a un psicoanálisis del conocimiento objetivo. México: Siglo XXI.

Bourdie, P. y Wacquant, L. 1995. Invitación a una sociología reflexiva. México: Siglo XXI.

Briones, G. 1994. Incompatibilidad de paradigmas y compatibilidad de técnicas en ciencias sociales. Revista de Sociología 9: 25-33.

Cook, T. D. y Reinchardt, C. 1986. Métodos cualitativos y cuantitativos en investigación evaluativo. Madrid: Morata.

Dilthey, W. 1986. Introducción a las ciencias del espíritu. Madrid: Alianza Editorial.

Facuse, M. 2003. Una Epistemología Pluralista: El anarquismo de la ciencia de Paul Feyerabend. Cinta moebio 17: 35-67. 
Feyerabend, P. 1978. La ciencia en una sociedad libre. México: Siglo XXI.

Feyerabend, P. 1991. Diálogo sobre el conocimiento. Madrid: Cátedra.

Lakatos, I. 1981. Matemáticas, ciencia y epistemología. Madrid: Alianza.

Morin, E. 1999. Los siete saberes necesarios para la educación del futuro. Paris: Unesco Editorial.

Toledo, U. 1998. La epistemología según Feyerabend. Cinta moebio 4: 1-20.

Toledo, U. 2004. ¿Una epistemología del Trabajo Social? Cinta moebio 21: 30-44.

Wallerstein, I. 1997. Abrir las ciencias sociales. México: Siglo XXI.

Nagel, E. 1981. La estructura de la ciencia. Madrid: Paidos.

Recibido el 5 Ene 2009

Aceptado el 15 Mar 2009 